\title{
FATOR DE RESPOSTA DA PRODUÇÃO DO CAFEEIRO AO DEFICIT HÍDRICO EM CAMPINAS ${ }^{(1)}$
}

\author{
FLÁVIO BUSSMEYER ARRUDA ${ }^{(2)}$; MARCOS ALEXANDRE GRANDE ${ }^{(3)}$
}

\begin{abstract}
RESUMO
A quantificação da resposta do cafeeiro à disponibilidade de água é um importante fator na estimativa da produção e na análise da viabilidade da irrigação. Nesse sentido, as produções de café irrigado (Yi) e não irrigado (Yni) de um experimento conduzido por 16 anos em Campinas (SP), foram analisadas como diferenças relativas (Yi-Yni)/Yi e correlacionadas com o déficit hídrico, expresso pelos respectivos valores de evapotranspiração real, (ETRi-ETRni)/ETRi, com $\mathrm{R}^{2}=0,6417$. A supressão de cada mês do ano permitiu verificar sua importância relativa na correlação. Abril, maio, junho e julho mostraram importância na relação com a produção. Foi possível o ajuste do coeficiente de sensibilidade ou fator de resposta da produção do cafeeiro ao déficit hídrico, $\mathrm{Ky}$, conforme recomendação da FAO. O fator de resposta de produção (Ky) anual mostrou-se linearmente crescente com a idade da planta, indicando aumento quanto à sensibilidade ao déficit hídrico ao longo dos anos.
\end{abstract}

Palavras-chave: café, déficit hídrico, irrigação, Ky.

\section{ABSTRACT \\ COFFEE YIELD RESPONSE FACTOR AS RELATED TO THE WATER DEFICIT}

Coffee yield response to available water is a major factor on yield prediction and irrigation feasibility. In this way, results of irrigated (Yi) and non irrigated (Yni) yields of a 16-year field experiment with coffee plants carried out in Campinas, State of São Paulo, Brazil, were analyzed as relative differences of (Yi-Yni)/Yi and correlated to the relative difference of actual evapotranspiration, (ETRi-ETRni)/ETRi. By suppressing each month of the year in that correlation, it was observed that water deficit occurred in April, May, June and July were important to yield analysis. It was possible to adjust the sensitivity coefficient (Ky) or the yield response factor to water deficit, as recommended by FAO. The annual yield response factor $(\mathrm{Ky})$ showed a linear relation to plant age indicating an increase of plant sensitivity to water stress along the years.

Key words: coffee, water deficit, irrigation, Ky.

(1) Parte da tese apresentada pelo segundo autor ao Instituto Agronômico de Campinas para a obtenção do título de M.S. em Agricultura Tropical e Subtropical. Recebido para publicação em 3 de dezembro de 2001 e aceito em 30 de janeiro de 2003.

(2) Centro de Ecofisiologia e Biofísica, Instituto Agronômico, Caixa Postal 28, 13001-970 Campinas (SP). E-mail: farruda@iac.sp.gov.br

(3) JP Engenharia. 


\section{INTRODUÇÃO}

Reconhecidamente, o cafeeiro é afetado pela seca com a conseqüente redução da produção. A utilização de práticas de conservação da umidade do solo ou de irrigação podem ser formas de mitigar os problemas de deficiência hídrica e de incrementos à produção.

Para a introdução de novas práticas ou mesmo para se saber qual o impacto da ocorrência de secas nas lavouras de café, há forte necessidade de se quantificar tal efeito na produção.

Na impossibilidade de se ter experimentos regionais irrigados realizados em longo período (mais de 10 anos de produção), contrastando o efeito da restrição da água no solo, a utilização de modelos ou de equações preditivas é a forma mais viável de se estimar as perdas de produção.

Dentre os vários métodos disponíveis para a quantificação do efeito hídrico na produção, o uso do fator de resposta da produção ou índice de sensibilidade ao déficit hídrico (Ky) é o que vem recebendo destaque e recomendação pela FAO (Doorenbos e KASSAN, 1979).

Muitas pesquisas têm sido realizadas na determinação de $\mathrm{Ky}$, como para a cultura da batata (Bezerra, 1995) e da soja (Moraes et al., 1998). Algumas dessas informações são sumarizadas em Doorenbos e Pruitt (1975), Doorenbos e Kassan, (1979) e, mais recentemente, ALLEN et al. (1998), porém sem a menção para a cultura de café. PICINI et al. (1999), utilizando dados estimados da produção potencial de café e resultados de experimento de campo, obtiveram bons resultados de Ky para Mococa (SP), mas não para a região de Campinas (PICINI, 1998).

No presente trabalho, o comportamento do índice de sensibilidade ao estresse hídrico do cafeeiro foi investigado para Campinas (SP), com o objetivo de dar melhor compreensão da resposta à irrigação por esta cultura.

\section{MATERIAL E MÉTODOS}

Os dados básicos utilizados no presente estudo são provenientes de um experimento de campo, coordenado pelo Dr. Geraldo Barreto, realizado em Campinas (SP), na área experimental da Seção de Irrigação junto à barragem Monjolinho, no período de 1957 a 1975, do Núcleo Experimental de Campinas, do Instituto Agronômico (IAC).

O clima da região é do tipo "Cwa", conforme classificação de Köppen, e o solo classificado como Latossolo Vermelho Eutroférrico, argiloso, série Monjolinho, localizado em relevo plano a suave ondulado, profundo e bem drenado. Os resultados das análises físicas, químicas e de retenção de água no solo são mostradas no Quadro 1, realizadas pelas Seções de Irrigação e Drenagem, de Pedologia e de Seção de Fertilidade do Solo e Nutrição de Plantas, do IAC.

A cultivar utilizada no experimento foi Mundo Novo 379/19, plantada em 20 de novembro de 1957, no espaçamento de $3 \times 2 \mathrm{~m}$, com duas plantas por cova, em área de $1.080 \mathrm{~m}^{2}$ para cada parcela. $\mathrm{O}$ desenvolvimento da cultura, adubações e tratamentos fitossanitários seguiram as recomendações técnicas do IAC e foram realizados pela Seção de Café.

Quadro 1. Resultados da análise física e química do solo no experimento de irrigação do cafeeiro em Campinas (SP)

\begin{tabular}{|c|c|c|c|c|c|c|}
\hline \multirow{3}{*}{$\begin{array}{l}\text { Profundidade } \\
\mathrm{m}\end{array}$} & \multirow{2}{*}{ Argila } & \multirow{2}{*}{ Silte } & \multicolumn{4}{|c|}{ Areia } \\
\hline & & & \multicolumn{2}{|r|}{ Fina } & \multicolumn{2}{|c|}{ Grossa } \\
\hline & & & g. $\mathrm{kg}^{-1}$ & & & \\
\hline \multirow[t]{3}{*}{$0-50$} & 550 & 240 & & 100 & & \\
\hline & Matéria Orgânica & $\mathrm{pH}$ & $\mathrm{Al}^{+++}$ & $\mathrm{Ca}^{++}+\mathrm{Mg}^{++}$ & $\mathrm{K}^{+}$ & $\mathrm{P}$ \\
\hline & g. $\cdot \mathrm{kg}^{-1}$ & & $\overline{-}$ & $\mathrm{m} \cdot \mathrm{mol}_{\mathrm{c}} \mathrm{kg}^{-1}$ & - & 1 \\
\hline $0-25$ & 54 & 5,4 & 0,3 & 3,9 & 54 & 10 \\
\hline \multirow[t]{2}{*}{$26-50$} & 39 & 5,4 & 0,1 & 3,7 & 63 & 3 \\
\hline & $\begin{array}{l}\text { Capacidade } \\
\text { de Campo }\end{array}$ & $\begin{array}{l}\text { Ponto de } \\
\text { Murcha }\end{array}$ & \multicolumn{2}{|r|}{ Densidade } & \multirow{2}{*}{\multicolumn{2}{|c|}{$\begin{array}{c}\text { Água } \\
\text { Disponível } \\
\mathrm{mm}\end{array}$}} \\
\hline & $x_{2}$ & - & \multicolumn{2}{|r|}{ g. $\mathrm{cm}^{-3}$} & & \\
\hline $0-100$ & 27,9 & 20,1 & \multicolumn{2}{|r|}{1,13} & & \\
\hline
\end{tabular}


O experimento foi arranjado no campo em parcelas subdivididas com os tratamentos das parcelas principais, dispostas em blocos ao acaso com seis repetições. Nas parcelas foram aplicados os tratamentos de irrigação desde 1960 e nas subparcelas, os tratamentos de tipos de condução de copa após o ano de 1966 . Cada subparcela, no total de 6, possuía 30 covas consideradas úteis. No presente estudo, analisaram-se apenas os tratamentos de livre crescimento irrigados e não irrigados.

As irrigações foram realizadas pelo sistema de aspersão convencional sobre copa, quando consumido $70 \%$ da água disponível na camada do solo de 0-100 cm, monitorada por amostragem gravimétrica.

Para a avaliação da disponibilidade da água no solo, foram realizados os balanços hídricos diários. Calculou-se a evapotranspiração potencial (ETP) a partir dos dados de evaporação de superfície livre (Ev) do tanque enterrado IA-58 (Tosello, 1960) pela equação ETP $=\mathrm{Kp} . \mathrm{Kp} \mathrm{p}^{\prime}$.Ev, conforme recomendado por Doorenbos e PruitT (1977).

A evaporação desse tanque se assemelha ao tanque Colorado, portanto, o coeficiente $\mathrm{K}_{\mathrm{p}}{ }^{\prime}=1,0$. Nas condições de vento moderado e uma área gramada entre 40 e $70 \mathrm{~m}$ têm-se coeficientes variando de 0,7 a 0,95 . No cálculo da evapotranspiração, ajustou-se um coeficiente de tanque $K_{p}=0,7$, com base nos dados de evapotranspiração decendial do mesmo período determinada pelo método de THORnTHWAite e MATTER (1955).

O coeficiente de cultura adotado seguiu o critério da correção com a idade e de partição em virtude do molhamento da superfície do solo e do secamento do perfil de solo, conforme recomendado por AlLEN et al. (1998).

Foram adotados os seguintes valores de coeficiente de cultura basal, $K_{\mathrm{cb}}$ (Quadro 2), em função da idade da planta, em condições de pleno suprimento de água, isto é, até o consumo de $50 \%$ da água disponível na camada de $0-100 \mathrm{~cm}(44,1 \mathrm{~mm})$.

Quadro 2. Valores de coeficiente de cultura basal adotados para o cálculo da ETR no período de 1959 a 1975

\begin{tabular}{ll}
\hline Ano & $\mathrm{K}_{\mathrm{cb}}$ \\
\hline 2 & 0,2 \\
3 & 0,4 \\
4 & 0,6 \\
5 & 0,8 \\
6 em diante & 1,0 \\
\hline
\end{tabular}

O efeito do molhamento da superfície do solo foi considerado sempre que as chuvas fossem maiores que $10 \mathrm{~mm}$, calculada como chuva agrícola. Para tanto, adotou-se $\mathrm{Kc}=1$, exatamente no dia da ocorrência e no dia posterior.

Para a penalização da transpiração pelo efeito da seca, com base em ARRUDA et al. (2000), a partir de $50 \%$ do consumo da água disponível no solo, o Kc era reduzido linearmente desde o valor de Kcb (Quadro 2) até o valor nulo correspondente ao consumo de $100 \%$ da água disponível no solo.

\section{RESULTADOS E DISCUSSÃO}

O cafezal ao longo de dezesseis anos de produção apresentou forte variação entre as safras, demonstrando a influência da produtividade do ano anterior na safra seguinte. Essa variação prejudicou as análises estatísticas, optando-se por utilizar a produção bi-anual, para facilitar a visualização da variação da produção, conforme apresentado na figura 1.

As curvas da figura 1 apresentaram um formato típico do livre crescimento observado em lavouras de café no Estado de São Paulo. O cafeeiro atinge sua produtividade máxima sete anos após o plantio, decrescendo com a conseqüente competição entre as plantas.

Na atualidade, essa competição é minimizada pela aplicação das técnicas de decote e poda, permitindo que esse decréscimo seja mais lento. Em todos os biênios a produção irrigada foi igual ou superior à não irrigada.

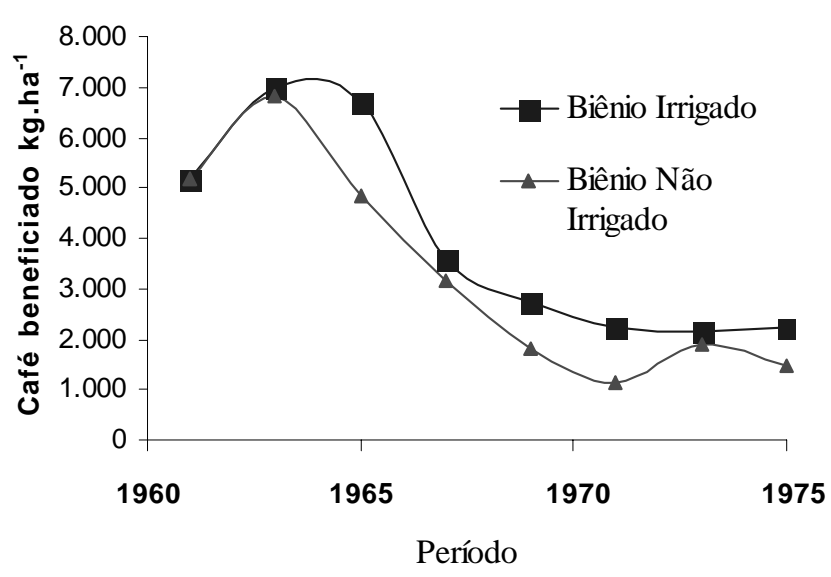

Figura 1. Média móvel da produção bi-anual de café $\left(\mathrm{kg} \cdot \mathrm{ha}^{-1}\right)$ para o tratamento irrigado e o não-irrigado, em Campinas (SP). 
Os resultados mostraram grande variação na produção e nas diferenças de produção entre o tratamento irrigado e o não-irrigado ao longo dos anos. A irrigação incrementou a produção em $347 \mathrm{~kg} \cdot \mathrm{ha}^{-1}$, com um máximo de $1.090 \mathrm{~kg} . \mathrm{ha}^{-1}$, apresentando variações devido à produção do ano anterior associado aos períodos de déficit hídrico.

Na figura 2, pode-se visualizar a variação da evapotranspiração potencial (ETP) no período de 1959 a 1975. A amplitude de variação foi de 1.024 a 1.429 $\mathrm{mm}$, demonstrando que neste período de cultivo, o consumo de água pelas plantas variou influindo no potencial produtivo a cada ano. Os valores de ETR foram sempre inferiores aos de ETP, mesmo no tratamento irrigado, somente se aproximando em 1972, quando apresentaram valores de 1.014 e $1.024 \mathrm{~mm}$ respectivamente. A evapotranspiração real no tratamento não irrigado (ETRni) não se igualou em nenhum ano aos valores de ETP ou mesmo à evapotranspiração real do tratamento irrigado (ETRi). Os valores da ETRni do tratamento não irrigado somente se aproximaram da ETP e ETRi do tratamento irrigado no período de 1971 a 1973.

Considerando-se que as maiores produções são obtidas quando não há restrições para a transpiração, então essa situação ocorreu somente de 1972 a 1973. O ano de 1968 foi o de maior deficiência, tendo a ETRni atingido $74 \%$ da potencial.

É conhecido o fato que as secas afetam a produção do cafeeiro. Porém, vários autores têm encontrado dificuldade em relacionar significativamente a produção com a deficiência de água ou precipitações (IAFFE et al., 2001). Tal fato é devido principalmente à alternância de produção do cafeeiro, ao padrão de produtividade ascendente e descendente ao longo da vida da cultura (Figura 1), da ocorrência simultânea do crescimento vegetativo e reprodutivo e por serem esses sorvedouros de assimilados internos da planta concorrentes entre si.

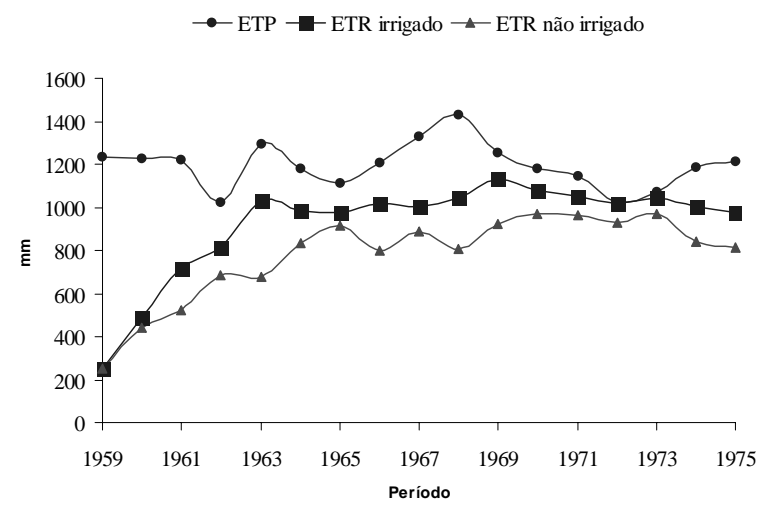

Figura 2. Variação da evapotranspiração potencial (ETP) e evapotranspiração real (ETR) do tratamento irrigado e o não-irrigado de cafeeiro em Campinas.
$\mathrm{Na}$ tentativa de melhor expressar o efeito hídrico na produção, investigaram-se várias correlações entre as diferenças de produtividade entre tratamento irrigado e não irrigado e os dados de precipitação (P), evapotranspiração potencial (ETP), evapotranspiração real irrigada menos a não irrigada (ETRi-ETRni), evapotranspiração relativa (ETRiETRni)/ETP, deficiência de água no solo e déficit hídrico pelo balanço de THORNTHWAITE e MATTER (1955). $O$ melhor resultado foi obtido entre a diferença relativa de produção, (Yi-Yni)/Yi, e a diferença relativa de evapotranspiração, (ETRi-ETRni)/ETRi.

As correlações foram investigadas ao longo do ano agrícola em vários períodos. Aquele que apresentou o melhor ajuste foi o compreendido entre abril do ano anterior e março do ano em que a colheita é realizada a partir de maio. Esse período é condizente com a seqüência dos estádios fenológicos para a produção da cultura (CAMARGO et al., 2001).

Os resultados obtidos da diferença relativa de produção em vista da diferença relativa de evapotranspiração entre o tratamento irrigado e o nãoirrigado, ocorrida no período de abril do ano anterior a março do ano da colheita do café, são apresentados na figura 3 e no quadro 3.

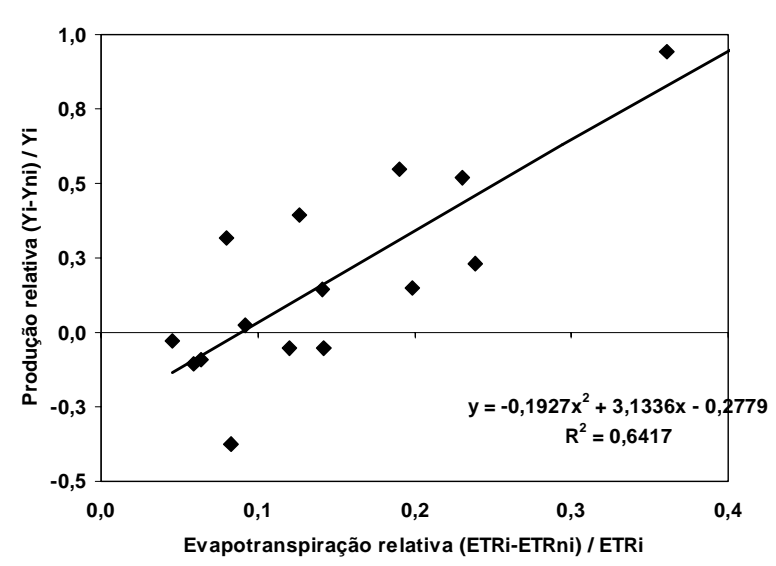

Figura 3. Produção relativa $(Y i-Y n i) / Y i$ em função da evapotranspiração relativa (ETRi-ETRni)/ETRi de tratamentos irrigado (i) e não irrigado (ni) da cultura do café em Campinas.

A correlação obtida na figura 3 apresentou $\mathrm{R}^{2}=64 \%$, da redução da produção com a redução na evapotranspiração do cafeeiro. A existência de anos com produções do tratamento irrigado pouco menores do que o não-irrigado resultou em alguns valores da diferença relativa de produção também negativos. A inclinação na curva apresentada na figura 3 indica uma aceleração na redução na produção com o aumento do déficit hídrico. 
Por exemplo, em 1960/61 uma diminuição leve na ETR da ordem de $9 \%$ resultou em uma redução na produção de apenas 2,5\%; em 1964/65 a redução média em ETR foi de $14 \%$ refletindo uma diminuição de $14,2 \%$ na produção. Acentuando-se a redução em ETR, acentua a redução na produção, como em 1968/69, em que $23 \%$ em ETR promoveu uma produção do nãoirrigado $52 \%$ menor que o irrigado.

A fim de avaliar a importância do efeito hídrico de cada mês em relação à produção, utilizouse da correlação apresentada na figura 3, porém, sistematicamente abstraindo-se do cálculo cada mês do ano. Os resultados são apresentados na figura 4.

Os valores das correlações na figura 4 , de modo geral, se mantiveram muito estáveis ao longo do ano, um indício de que a redução na ETR do cafeeiro durante todo o ano afeta a produção da lavoura. O mês menos sensível foi agosto e os mais sensíveis foram abril, maio, junho e julho. Agosto é o que apresenta menor sensibilidade ao déficit hídrico durante o período de seca, em Campinas.

Aparentemente, agosto deve ser o mês indicado para a suspensão da irrigação para a indução do florescimento no cafeeiro. CARR (2001), em ampla revisão sobre o assunto, descreveu não ser necessária a irrigação nesse período de dormência, e considera até mesmo benéfico um período de estresse hídrico para uniformização da florada do cafeeiro. Essa assertiva é hoje bem aceita por vários técnicos do setor, porém, deve ficar claro que pode haver alguma redução na produção, conforme resultados de CAMARGO et al. (1984), utilizando uma barcaça como impedimento para a precipitação em diferentes períodos no cafeeiro, em Campinas.

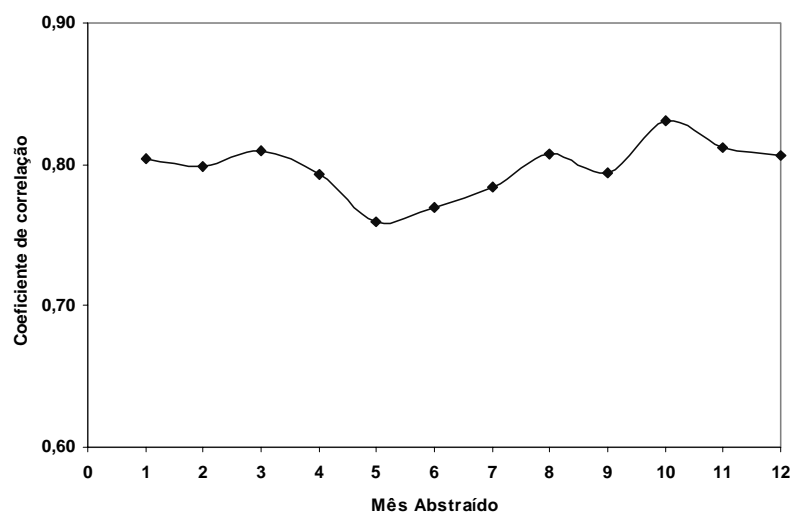

Figura 4. Valores do coeficiente de correlação entre (YiYni)/Yi versus (ETRi-ETRni)/ETRi, calculado sem a inclusão de cada mês do ano, em 16 anos de experimento em Campinas.

A modelagem do efeito da deficiência hídrica na produção tem-se valido da relação [(Yi-Yni)/Yi] / [(ETRi-ETRni $) /$ ETRi $]=\mathrm{Ky}$, conforme proposto pela FAO (Doorenbos e Kassan, 1979; Allen et al., 1998). Os componentes para o cálculo de Ky e os resultados obtidos a cada ano agrícola são apresentados no Quadro 3. A amplitude de variação observada de Ky foi desde $-4,547$ até 3,951 .

Quadro 3. Resultados de Ky obtidos no período de abril a março de cada ano, calculados a partir da diferença relativa entre as produções do tratamento irrigado (Yi) e do nãoirrigado (Yni) e das diferenças relativa entre as evapotranspiração real do irrigado (ETRi) e do não-irrigado (ETRni), em 16 anos de experimento em Campinas (SP)

\begin{tabular}{|c|c|c|c|c|c|c|}
\hline Ano & $\mathrm{Yi}$ & Yni & Yi-Yni & $\frac{\left(Y_{i}-Y_{n i}\right)}{Y i}$ & $\frac{(\text { ETRi-RTNi) }}{\text { ETRi }}$ & Ky \\
\hline \multicolumn{7}{|c|}{ kg.ha-1 } \\
\hline $59 / 60$ & 2.077 & 2.133 & -56 & -0.027 & 0.045 & -0.597 \\
\hline $60 / 61$ & 3.109 & 3.031 & 78 & 0.025 & 0.092 & 0.272 \\
\hline $61 / 62$ & 1.894 & 1.454 & $440^{*}$ & 0.232 & 0.238 & 0.974 \\
\hline $62 / 63$ & 5.103 & 5.383 & -281 & -0.055 & 0.142 & -0.386 \\
\hline $63 / 64$ & 1.159 & 69 & $1.090^{*}$ & 0.941 & 0.361 & 2.604 \\
\hline $64 / 65$ & 5.548 & 4.760 & $788^{*}$ & 0.142 & 0.142 & 1.002 \\
\hline $65 / 66$ & 422 & 460 & -38 & -0.090 & 0.064 & -1.403 \\
\hline $66 / 67$ & 3.149 & 2.681 & 468 & 0.149 & 0.199 & 0.748 \\
\hline $67 / 68$ & 864 & 911 & -47 & -0.054 & 0.120 & -0.451 \\
\hline $68 / 69$ & 1.876 & 900 & $976^{*}$ & 0.520 & 0.231 & 2.256 \\
\hline $69 / 70$ & 1.359 & 614 & $745^{*}$ & 0.548 & 0.191 & 2.873 \\
\hline $70 / 71$ & 867 & 523 & $343^{*}$ & 0.396 & 0.126 & 3.137 \\
\hline $71 / 72$ & 1.030 & 1.139 & -109 & -0.106 & 0.059 & -1.796 \\
\hline $72 / 73$ & 1.106 & 755 & $352^{*}$ & 0.318 & 0.080 & 3.951 \\
\hline $73 / 74$ & 182 & 251 & -68 & -0.375 & 0.082 & -4.547 \\
\hline $74 / 75$ & 2.059 & 1.233 & $826^{*}$ & 0.401 & 0.150 & 2.681 \\
\hline
\end{tabular}

$\left(^{*}\right)$ Diferença significativa a $5 \%$ pelo teste $t$. 
Esses valores são muito mais amplos do que os relatados na literatura para citros (ALLEN et al., 1998), e para café por PICINI et al. (1999) e por CARR (2001). A dificuldade de obtenção de valores de ETR, os resultados escassos na literatura sobre Ky em culturas perenes, bem como as diferenças com os aqui observados, indicam a necessidade de mais investigações nessa área de trabalho.

Os valores negativos de Ky são resultados da ocorrência de algumas produções do tratamento irrigado serem menores do que o não-irrigado. A alternância de produção provoca efeitos negativos da aplicação da irrigação, desaparecendo quando se avalia a produção bienal. Em nenhum desses casos, porém, houve significância na diferença de produção.

Uma importante informação observada no quadro 3 é que para os valores de Ky igual ou menor a 0,748 não houve diferença significativa entre as produções do cafeeiro. Trata-se do período de 1966/67, quando houve diferença de produção de $468 \mathrm{~kg} \cdot \mathrm{ha}^{-1}$; no entanto, ela não foi significativa devido à grande variação dentro do tratamento. Todas as produções observadas do tratamento irrigado foram significativas quando Ky era pelo menos igual a 0,974.

Os melhores resultados obtidos de Ky, isto é, nos anos significativos de aumento de produção devido à irrigação, estão indicados na figura 5 .

$\mathrm{O}$ ajuste linear crescente apresentado nessa figura indica a tendência de aumento de Ky com a idade da planta. Tal fato é indicador de que a produção do cafeeiro se torna mais sensível à redução da ETR ao longo dos anos.

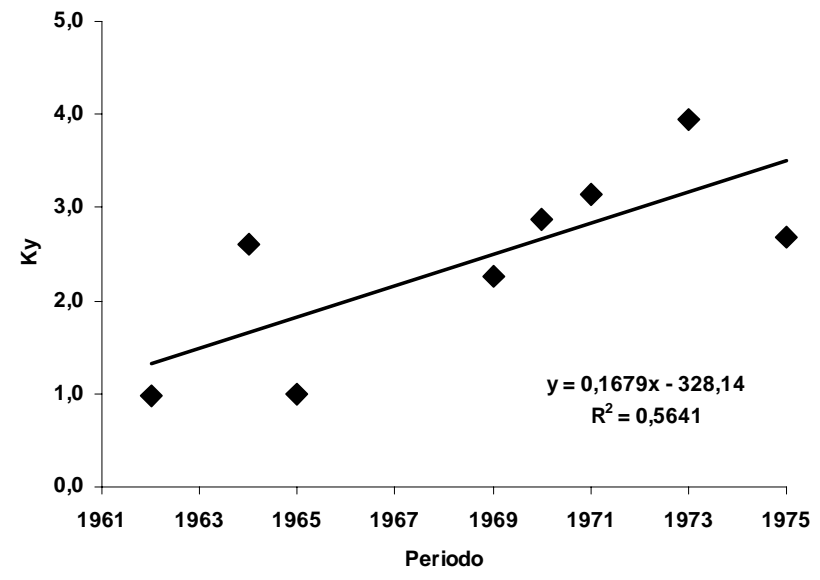

Figura 5. Resultados de Ky obtidos no período de abril a março de cada ano com diferença significativa entre as produções do tratamentos irrigado e do não-irrigado.

\section{CONCLUSÃO}

1. O índice de sensibilidade, $\mathrm{Ky}$, e a diferença relativa das produções em função da diferença relativa entre a evapotranspiração de tratamento irrigado e não irrigado mostraram-se bons indicadores para avaliação do déficit hídrico no cafeeiro.

2. No ano-calendário, maio mostrou-se o mais importante quanto à redução da ETR afetando a produção do cafeeiro, e agosto foi o menos importante e plausível de suspensão da irrigação.

3. Valores crescentes de Ky indicaram o aumento da sensibilidade do cafeeiro ao déficit hídrico com a idade da cultura.

4. Diferenças significativas de produtividade do cafeeiro devido à irrigação foram obtidas sempre que Ky foi maior que 0,974, em 16 colheitas.

\section{REFERÊNCIAS BIBLIOGRÁFICAS}

ALLEN, R. G.; PEREIRA, L. S.; RAES, D; SMITH, M. Crop evapotranspiration: guidelines for computing crop water requirements. Rome: Food and Agriculture Organization of the United Nations, 1998.300p. (Irrigation and Drainage, Paper 56)

ARRUDA, F. B.; IAFFE, A.; SAKAI, E,; CALHEIROS, R. O. Resultados anuais do coeficiente de cultura do cafeeiro em um ensaio em Pindorama, SP. In: SIMPÓSIO BRASILEIRO DE PESQUISA EM CAFEICULTURA IRRIGADA, 2000, Araguari. Anais... Araguari: Núcleo de Cafeicultura Irrigada/PNP\&D/ Embrapa Café, 2000 e SIMPÓSIO DE CAFÉS DO BRASIL, 1., 2000, Poços de Caldas. Anais... Brasília: Embrapa Café/Minas Plan, 2000. p.790-793.

BEZERRA, F.M.L. Coeficientes de cultura e efeitos de déficits hídricos nos diferentes estádios fenológicos sobre a produção da batata (Solanum tuberosum L.). 1995. 131p. Tese (Doutorado) - Escola Superior de Agricultura "Luiz de Queiroz", Piracicaba.

CAMARGO, A.P; GROHMANN, F.; DESSIMONI, L.M.; TEIXEIRA, A.A. Efeitos na produção do café de épocas de rega e de supressão da água por meio de cobertura transparente (barcaça). In: CONGRESSO BRASILEIRO DE PESQUISAS CAFEEIRAS, 1984, Londrina. Anais... Londrina: IBC, 1984. p.62-64.

CAMARGO, A.P.; CAMARGO, M.B.P.; PALLONE FILHO, W.J. Modelo climático-fenológico para determinação das necessidades de irrigação de café arábica na região Norte de São Paulo e no Triângulo Mineiro. Campinas: Instituto Agronômico, 2001. 26 p. (Boletim técnico IAC, 190)

CARR, M.K.V. The water relations and irrigation requirements of coffee. Experimental Agriculture, Cambridge, v. 37, p.1-36, 2001. 
DOORENBOS, J.; PRUITT, W.O. Guidelines for predicting crop water requirements. Rome: Food and Agriculture Organization of the United Nations, 1975. 179p. (Irrigation and Drainage, Paper 24)

DOORENBOS, J.; KASSAM, A.H. Yield response to water. Rome: Food and Agriculture Organization of the United Nations, 1979. 193p. (Irrigation and Drainage, Paper 33)

IAFFE, A.; ARRUDA, F B.; SAKAI, E.; PIRES, R.C.M.; QUAGLIA, L.; CALHEIROS, R.O. Avaliação de variáveis agrometeorológicas na produtividade de café em Campinas, SP. CONGRESSO BRASILEIRO DE ENGENHARIA AGRÍCOLA, 30, 2001, Foz do Iguaçu, 2001. Anais... Foz do Iguaçu: Sociedade Brasileira de Engenharia Agrícola, 2001. (CD-Rom)

MORAES, A.V.C.; CAMARGO, M.B.P.; MASCARENHAS, H.A.A.; MIRANDA, M.A. C.; PEREIRA, J.C.V.N.A. Teste e análise de modelos agrometeorológicos de estimativa de produtividade para a cultura da soja na região de Ribeirão Preto. Bragantia, Campinas, v.57, n.2, p.393-406p, 1998.
PICINI, A.G. Desenvolvimento e teste de modelos agrometeorológicos para a estimativa de produtividade do cafeeiro (Coffea arabica L.) a partir do monitoramento da disponibilidade hídrica do solo. 1998. 132p. Tese (Mestrado) - Escola Superior de Agricultura "Luiz de Queiroz", Piracicaba.

PICINI, A.G.; CAMARGO, M.B.P.;ORTOLANI, A.A.;FAZUOLI, L.C.; GALLO, P.B. Desenvolvimento e teste de modelos agrometeorológicos para a estimativa de produtividade do cafeeiro. Bragantia, Campinas, v.58, n.1, p.157-170, 1999.

THORNTHWAITE, C.W.; MATHER, J.R. The water balance. Centerton: Drexel Institute of Technology, Laboratory of Climatology, 1955. 104p. (Publications in Climatology, v. 8, n. 1)

TOSELLO, R. N. Novo tipo de evaporímetro terrestre. Bragantia, Campinas, v.19, p.731-751, 1960. 